\title{
Neuropsychologic Testing in Chiasmal Patients Exhibiting Inattention in the Temporal Visual Space during Monocular Visual Testing
}

\author{
Hans C. Fledelius ${ }^{1 *}$, Hanne Udesen ${ }^{2}$ \\ ${ }^{1}$ Eye Clinic, Rigshospitalet, Copenhagen University, Copenhagen, Denmark \\ ${ }^{2}$ Neurology Department, Section Neuropsychology, Rigshospitalet, Copenhagen University, Copenhagen, Denmark \\ Email: `hcfled@mail.dk
}

How to cite this paper: Fledelius, H.C. and Udesen, H. (2017) Neuropsychologic Testing in Chiasmal Patients Exhibiting Inattention in the Temporal Visual Space during Monocular Visual Testing. Open Journal of Ophthalmology, 7, 21-30.

https://doi.org/10.4236/ojoph.2017.71004

Received: November 14, 2016

Accepted: January 31, 2017

Published: February 3, 2017

Copyright $\odot 2017$ by authors and Scientific Research Publishing Inc. This work is licensed under the Creative Commons Attribution International License (CC BY 4.0).

http://creativecommons.org/licenses/by/4.0/

\begin{abstract}
Objective: By means of neuropsychologic tests, to further analyse a specific chiasmal monocular visual testing behaviour, here labelled temporal blocking because of the elective ignorance of optotypes on the temporal side of the chart. Often it is combined with impairment of reading and other cognitive impairments. Methods. Eighteen patients with lesions to the chiasm and some degree of temporal blocking aged 24 - 76 years underwent: 1) tests for visual neglect (Gothenburg test; behavioural inattention tests: star cancellation; line bisection); 2) visuo-perceptual tests; and 3) a test involving reading a crowded ten-letter and cipher bar. Results. The temporal blocking in two patients recovered after emergency neurosurgery and their results were normal when subsequently tested. Of the 16 patients with deficiencies, 14 had a poorer left eye $(\mathrm{p}<0.01)$. Conclusions. The best neuropsychologic tests appeared to be those for visual neglect and the crowded bar test. In most cases, the right cerebral hemisphere's lack of some crossed information from the left eye, usually needed for normative saccades and adjustment to visual space, may be a factor underlying the specific visual behaviour.
\end{abstract}

\section{Keywords}

Lesions to the Optic Chiasm, Visual Blocking to the Temporal Side, Neglect-Like Behaviour, Neuropsychologic Testing

\section{Introduction}

With neuro-ophthalmologists as the target group, we published reports on our first four chiasmal patients in whom the specific lateralizing monocular visual acuity testing behaviour was recognized with an apparent blocking of visual at- 
tention to the temporal side on the chart [1]. With more than 50 cases subsequently observed, unilateral or bilateral, the apparent not infrequent occurrence as part of chiasma-related pathology was stressed and the clinical diversity has been further elaborated in more recent papers [2] [3]. Apart from a brief mention of monocular visual chart testing behaviour [4], textbooks are usually reticent on the issue.

The chiasmal patients selected for the above studies all had some degree of a neglect-like pattern showing lateralizing inattention in visual space at customary clinical monocular visual acuity testing, in one or both eyes. Contrary to neglect per se, the patients included were fairly well aware that there was a full line, but could only capture the optotypes nasally on the chart/screen. The symbols to the temporal side appeared hidden and were ignored, despite the examiner's repeated request to have them all reported, and temporal blocking was suggested as a clinical term. Figure 1 illustrates absolute and graded blocking.

Some patients could hardly read a text, often quite out of proportion to the visual acuities and/or field defects recorded; a few patients also had cognitive problems in visual space. Mainly, however, the specific visual loss was partly compensated when seeing with both eyes, and the standard patient was not precise when asked about visual problems. In a small number, the visual problems increased after surgical removal of the lesion to the chiasm, typically a pituitary adenoma, but in most cases there was a definite improvement, or even a full return to normal visual function.

With neglect-like features as a keyword, we tentatively applied neuropsychologic testing to some of the patients. In oral presentations, we had previously
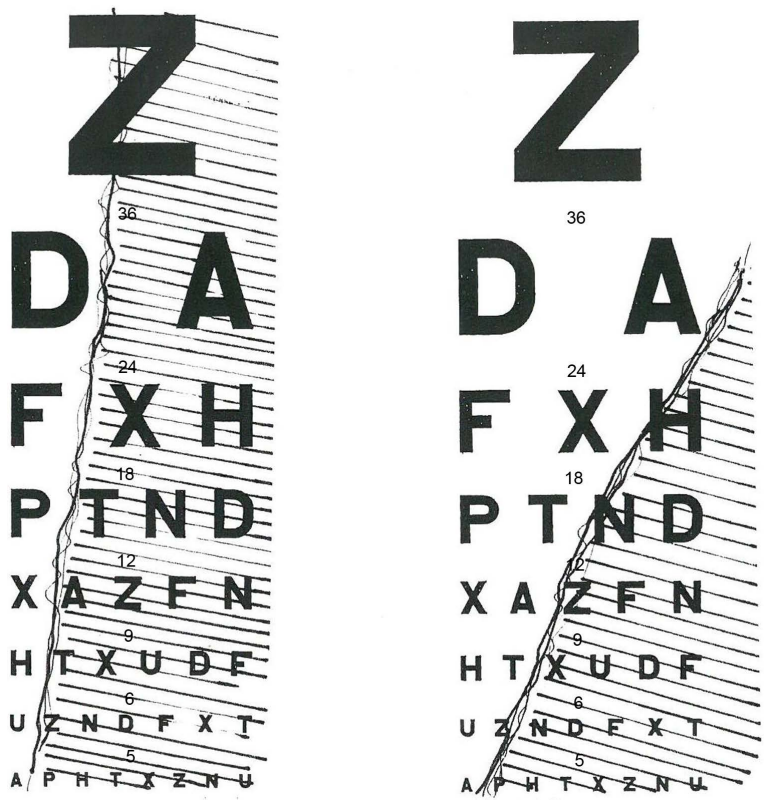

Figure 1. Right eye visual acuity testing showing absolute temporal blocking (left) and graded blocking (right). The ignorance in the temporally located field may comprise a larger or smaller part of the chart, and may also depend on how small the optotypes are (graded blocking). 
discussed preliminary results from single cases (SOE'99, EUNOS 2005), with a focus on why the patients do not move their eyes and miss part of the visual space. For the present paper, we have condensed our updated, more systematic experience, here based on 18 patients. Our aim has been to outline and test a neuropsychologic programme applicable to such patients, possibly to throw light on the underlying pathogenetic mechanisms relating to the visual pathway and its further connections.

\section{Methods}

Neuropsychologic testing was performed on 18 patients during 1999-2007 as a supplement to current ophthalmic evaluation. Seven were female, age $24-72$ years, median age 49 years. Eleven were male, age 38 - 76 years, median age 55 years. In two patients, the specific visual problem occurred after a traffic accident resulting in brain concussion. Sixteen had surgery for tumours adjacent to the chiasm: 13 for pituitary adenoma, and three for craniopharyngioma. The clinical data are specified in Table 1 in chronological order. All patients gave informed consent, and the study respected the tenets of the Helsinki declaration.

The standard ophthalmic evaluation (by H.C.F.) comprised: monocular distance visual acuity testing of each eye, using a Snellen chart and/or projector optotypes, with best glasses in a trial frame, followed by binocular testing. Visual fields were tested by conventional tangent screen evaluation, by computerized static perimetry (many could not yield a satisfactory reliability factor), by kinetic Goldmann perimetry (usually fair compliance), and by Amsler chart for subjective central near vision field registration. Colour sense was tested by Ishihara's isochromatic plates; the two-ciphered presentations occasionally disclosed visual ignorance to the temporal side. Eye motility was assessed, as well as slit-lamp examination, Goldmann applanation tonometry, and funduscopy.

The neuropsychologic tests (by H.U.) were preceded by a questionnaire asking for the patients' conception of his/her visual problem.

Comparing frequencies, the Chi square and Fisher's exact probability tests were used.

\subsection{Traditional Neglect Tests}

In the visual scanning test, often called the Gothenburg test in Scandinavia [5], the visual search pattern of the patient is examined on an A4 sheet of paper with 32 letters and ciphers randomly located across the paper. The instruction is to read all the symbols, without using a finger or other kind of marker (Figure 2). This is usually done in less than 25 seconds.

Behavioural inattention tests (BIT) included two tests [6]:

1) Star cancellation consists of a random array of verbal and non-verbal stimuli: 52 large stars, 13 randomly positioned letters and 19 short printed words interspersed with 54 smaller stars. The patient is handed a pencil and asked to locate the small stars and cross them out. This is usually done in less than $40-50$ seconds with no omissions. 
Table 1. Clinical data of the 18 chiasmal patients who underwent neuropsychologic evaluation.

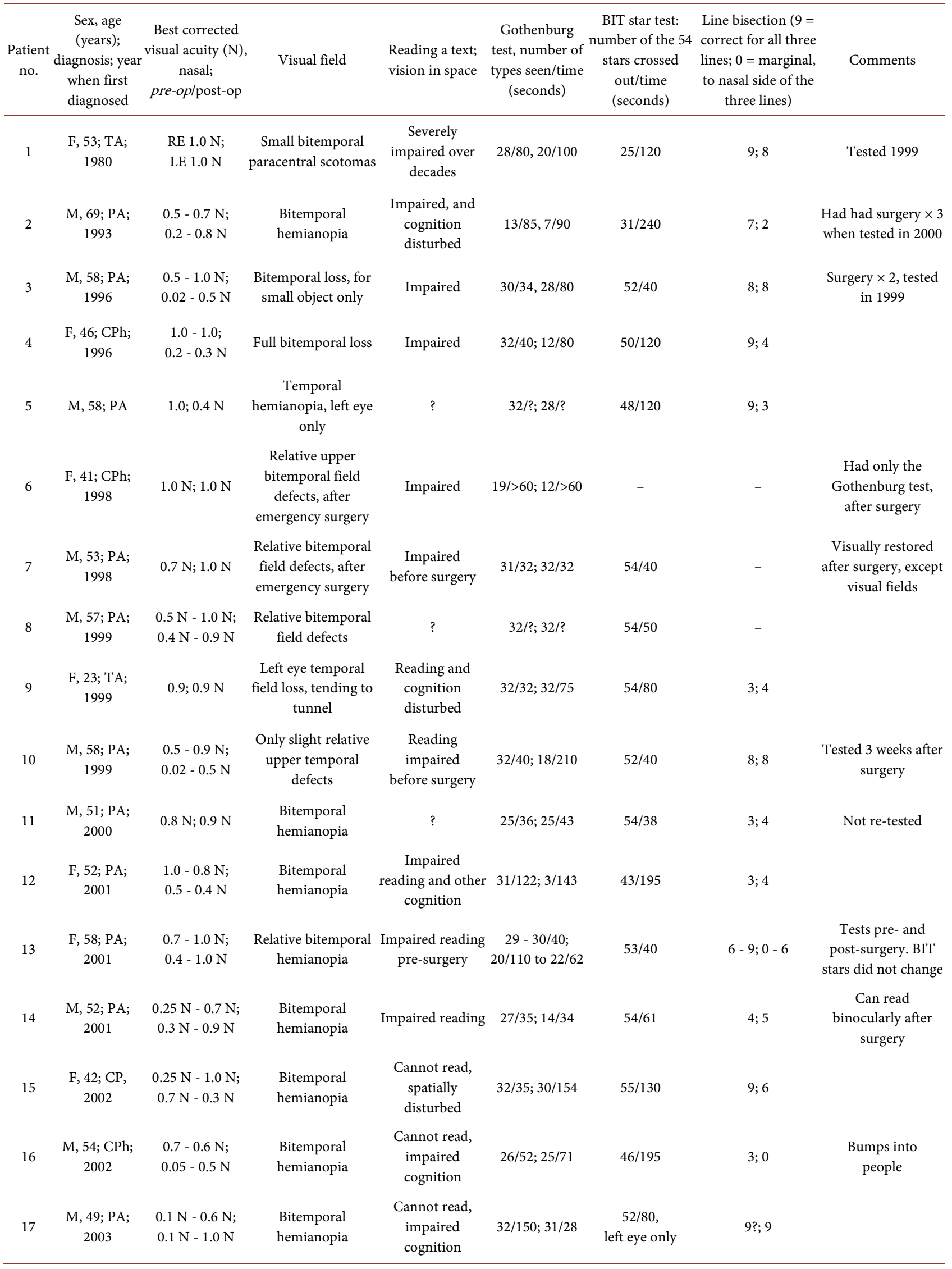




\section{Continued}

\begin{tabular}{ccccccc}
\hline & M, 37; CPh; & $0.5 \mathrm{~N}-0.5 \mathrm{~N} ;$ & Bitemporal & Reading + & & \\
2003 & $0.6 \mathrm{~N}-0.8 \mathrm{~N}$ & hemianopia & $\begin{array}{l}\text { cognition } \\
\text { impaired }\end{array}$ & $30 / 41 ; 25 / 28$ & $54 / 140$ & $8 ; 6$ \\
\hline
\end{tabular}

All patients underwent surgery for their tumours (PA, pituitary adenoma; $\mathrm{CPh}$, craniopharyngioma), except the two patients with skull trauma due to TA (traffic accident). The right eye best corrected visual acuity (RE) is given before the left eye acuity (LE) in decimal values. Two acuities are given per eye, initial and eventual, with initial vision italicized when before tumour removal. $\mathrm{N}$ after visual acuity (nasal seeing) indicates blocking or ignorance to the temporal side of the visual chart during monocular testing; $(\mathrm{N})$, only minimum temporal ignorance, although reproducible.
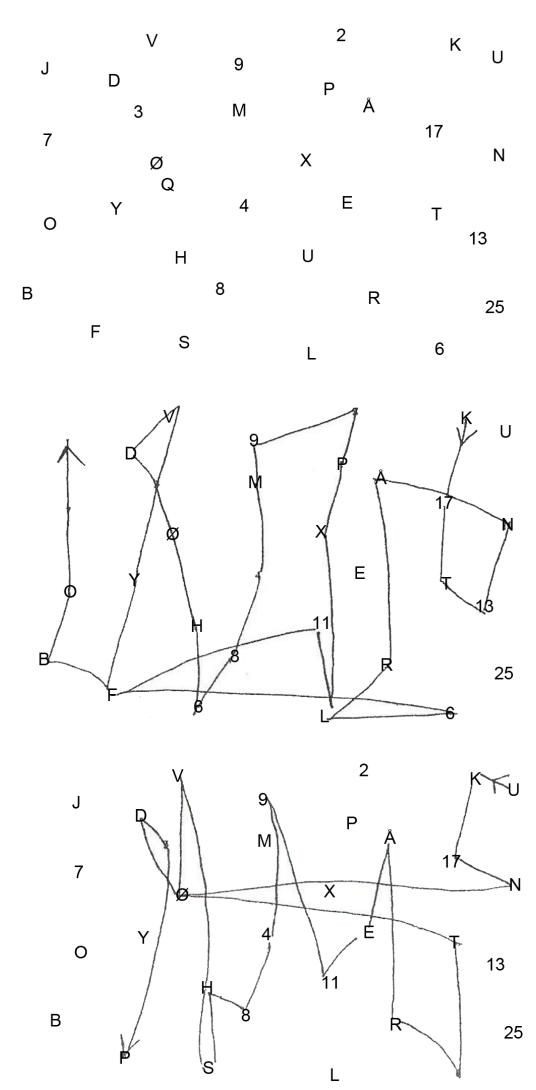

Figure 2. (Top) The Gothenburg test with 32 scattered ciphers and letters on an A4 sheet, to be read by best strategy. The performance of a chiasmal patient is shown for the right eye (centre), and for the left eye (bottom), with the sequence of the symbols perceived and recorded by the examiner. The right eye (visual acuity 1.0) saw all symbols but three in 35 seconds. The left eye (visual acuity 0.4 ) missed 12 of the 32 symbols, and the more haphazard performance took 110 seconds.

2) The line bisection test involves three horizontal black lines displayed in a staircase fashion across the page. Each line is pointed out to the patient, who is then instructed to the mark the centre. With part of the line ignored, typically to the temporal side, the mark will be skewed. The answers are graded from 3 (centre, correct) to 0 (nasal marginal) for each line, allowing a combined normal top score of 9 .

\subsection{Tests of Visuo-Perceptual Abilities}

Tests of visuo-perceptual ability are based on recognition of fragmented and degraded material and recognition of chimeric faces [7]. A test involving reading a 
crowded letter and cipher bar, with ten tightly packed symbols on a line, was used only in the late part of our series. Reaction time as an independent parameter was recorded at each test.

The correctness of the answers and the time required to complete the various tests were the main outcome parameters. The Gothenburg test and the line bisection test were carried out monocularly, preferably with the poorer eye first, then the better eye, and finally the test was repeated for the poorer eye. The eye should now have learnt the lesson, but drop-outs were usually repeated at re-testing. The remaining tests were done using both eyes.

The original visual pathway lesions were diagnosed one week to 19 years earlier. In each case an updated neuroophthalmological status was achieved just before and after the neuropsychological testing.

\section{Results}

Two of the 18 patients initially had emergency surgery as a result of alarming neuro-imaging combined with marked visual loss, including temporal blocking. Both patients first underwent their neuropsychologic tests shortly after uneventful neurosurgery, and they achieved normal results. In the context, they may be regarded controls for the remaining 16 patients, all of whom missed symbols or interpretations, and/or required abnormally a long time to do the tests.

Evaluating the monocularly conducted Gothenburg test, the 32 eyes of the 16 patients could be subdivided into 27 with some degree of temporal blocking on the visual chart and the five fellow eyes that appeared normal in this respect. In the blocking subgroup, the median values were 25 correct indications (range, 12 - 32) versus a range of 25 - 32 in the non-blocking eyes, and distributions differed significantly ( $\mathrm{p}<0.01$ by Kolmogorov-Smirnov and $\chi^{2}$ test). The same held for the time required for the Gothenburg test, with median scores of 80 seconds and 33 seconds in the two subgroups, respectively. The binocularly performed BIT test showed no statistical difference between patients who had blocking of both eyes versus those with only one eye affected.

Comparing right versus left eyes, two of the 16 had a poorer performance in the right eye. In both cases, this was associated with a significant visual acuity loss in that eye. The remaining 14 had a poorer performance in the left eye. This predominance of the left eye was statistically significant ( $p<0.01$, binomial test). In five of the 14 patients, more visual loss in the left eye might be at least partly responsible. In the remaining nine patients, acuity measured on the Snellen chart was similar, although with poorer performance in the left eye maintained during the neuropsychologic evaluation.

As a clinical supplement to Table 1, four cases are described in more detail, arranged according to increasing visual incapacity.

\subsection{Case 1}

A 58-year-old male university administrator (patient 10, Table 1) had experi- 
enced loss of words in a text and increasing reading difficulty, despite corrected acuities of 1.0 and 0.4 and only marginal affection of visual fields.

In pre-operative testing, for the Gothenburg test his left eye missed 14 symbols and needed 210 seconds to complete the test, whereas the right eye saw all 32 symbols and required 40 seconds. On re-testing the affected left eye, by means of compensating strategies he managed 27 of the 32 points, now in only 55 seconds. Line bisection was almost precise with each eye tested. On the BIT test, using both eyes, he missed only two of the 54 small stars. He recovered visually within a few days after surgery for pituitary adenoma and subsequently passed the repeated tests without errors and at a speed close to normal.

\subsection{Case 2}

A woman aged 72 years (patient 1, Table 1) had been in a traffic accident resulting in brain concussion two decades ago. Her clinical details were given in a previous paper [1]. Although she had only minute permanent paracentral bitemporal scotomas, she could identify only the outer nasal column on the Snellen chart with each eye separately, symmetrically down to acuity 1.0 (absolute temporal blocking, $80 \%$ of the chart completely lost), but full lines when using both eyes. Binocular reading of a text had been slow and difficult since the accident.

When neuro-psychologically tested 19 years after the accident, her left and right eyes could see 17 and 28 symbols, which increased to 23 and 31 - 32 symbols on re-testing, respectively, but still required about 90 seconds. Her line bisection was fair, but she found only 25/54 of the BIT stars using both eyes and taking two full minutes. She also had significantly reduced binocular recognition of degraded picture material.

\subsection{Case 3}

A 38-year-old man (patient 18, Table 1) had surgery for a pituitary adenoma. One year earlier he had been referred for acuities 0.8 and 0.9 and operated on for some exophoria trouble, the likely chiasmal basis for which was not then recognized. Eventually his symptoms worsened, in particular regarding reading ability, and his tumour was diagnosed.

When tested 2 weeks after surgery, he had acuities of $<0.5$ and 0.6 in the right and left eyes, respectively. Both eyes showed typical temporal visual blocking at monocular visual acuity testing, whereas reading a text remained impaired. Outside the hospital, he could not recognize previously familiar geographic locations, his local railway station for instance.

His Gothenburg chart test found all 32 symbols for the left eye, when repeated, and 30 and 32 correspondingly for the right eye, and at a speed coming close to normal (41 and 28 seconds, respectively). Crossing out the BIT stars took three times the normal time, and his line bisections were slightly nasally skewed (scores 8 and 6).

\subsection{Case 4}

A 52-year-old female laboratory worker (patient 12, Table 1) had severe visual 
disorientation and massive bitemporal hemianopia after surgery for a huge pituitary adenoma. Pre-operatively, with acuities of 1.0 and 0.5 , she performed much better, and there was only a slight bitemporal visual field loss.

When tested 4 weeks post-operatively, acuity in her right eye was $<1.0$ and temporally blocked (graded) $0.4-0.5$ acuity in the left eye. After 143 seconds, she found only three of the 32 Gothenburg chart symbols with her left eye; she saw $31 / 32$ symbols with the right eye, but also required extra time (122 seconds). For the binocular BIT tests, she took 195 seconds to cross out 43 of the 54 stars. Line bisection was skewed, away from the blocked part, and she had the lowest score for a supplementary drawing test involving copying simple figures. She can hardly read a printed text, and in virtual space among people she has remained confused.

\section{Discussion}

Although not rare in patients with chiasmal lesions, the specific monocular visual testing behaviour under study has not gained much medical attention. One obvious reason is that typically the chiasmal patient is vague regarding visual complaints because there is often everyday compensation for monocular dropouts when using both eyes. Often, blurred clues will underlie a combined patient + doctor delay.

Diagnostic inertia may be further compounded by the obvious difficulty in explaining (and accepting) not only the rather specific visual pathway drop-outs at visual testing but also the related consequences for the overall visual abilities encountered in some patients. Clearly, there seems to be neglect-like features, although in the acknowledged absence of a relevant suprageniculate lesion [8] [9] [10]. Further, contrary to ignorance about the perceptual disability in real neglect, the chiasmal patient with temporal blocking is aware that something is very wrong when totally failing on the temporal side of the visual chart. A few can compensate marginally by peculiar head turns or voluntary eye movements, a situation, however, so very far from the saccadic visual search mechanisms that automatically come into play when normally coping with the visual world around.

Neuropsychologic testing was performed as a supplement in 18 of our original 50 patients, in an attempt to better understand the deviant visual patterns observed during the ophthalmic evaluation. With no established testing set-up for the visual symptom complex under study, we applied various tests and strategies in order to gain experience. From our present analysis, we would now recommend the three neglect tests (Gothenburg chart; BIT star cancellation; line bisection) and the crowded bar test (or a similar reading test). Preferably, the tests should be carried out for each eye, the poorer before the better performing eye, and for both eyes together. We also found it useful to re-test the poorer eye, after the patient had confronted the initial shortcomings, but usually there was no significant improvement. As illustrated by the time factor, testing was tiring, or even painful for some. In practical terms, this set a limit on what could be in- 
cluded in a single testing session. In the full context, we would use the visuoperceptual tests (degraded pictures; chimeric faces) mainly as a diagnostic supplement in those with least problems. In general, practical hints and compensations could sometimes be suggested based on the neuropsychologic testing, and the patients might also find mental back-up in their often awkward social situation.

We are left with the question regarding the mechanisms underlying the peculiar perceptual behaviour of visual space inattention, or blocking to the temporal side, as occasionally observed in chiasmal patients. On a more general level, one might hypothesize that some visual input, usually required for automatically moving the eye in space, has been lost. Saccades relevant for current fixation are not released, and there will be visual inattention with apparently blinded sectors. Further, if parvocellular axons are more vulnerable to tumour pressure than magnocellular axons, the stratification of messages sent on to the suprageniculate central nervous system structures might be curtailed and some higher elaboration in a programmed sequence of conductional events might suffer.

With clinical features suggestive of neglect, the focus is on the non-dominant hemisphere [8] [9] [10]. Among the 16 patients with a side difference in overall testing, the left eye had the poorer performance in 14 patients $(\mathrm{p}<0.01$, binomial test). With temporal visual field defects due to nasal retinal perceptual loss, this implies a reduction of crossed visual information to the right hemisphere, where malfunction due to organic lesions traditionally may underlie real neglect. Reduced visual acuity, however, might have contributed to the side difference. Excluding the two right eyes and five left eyes where this was the case, there remained nine patients with only a small side difference in visual acuity. When tested, the left eye was worse in all 9 ( $\mathrm{p}<0.01$, binomial test), possibly suggesting some right hemisphere deprivation of essential crossing of visual information in space, on which it would normally react. We should add here that a magnetic resonance imaging brain scan was performed in all patients and showed no evidence of lesions other than those of anatomic relevance for the basic pathology related to the optic chiasm. Theoretically, a functional positron emission tomography scan could throw light on active versus missing brain activity in relevant brain domains [11], but a subsequent small-scale study has not provided further understanding [12].

\section{Conclusion}

The objective remains to increase the awareness of clinicians when occasionally observing visual inattention on the temporal side in single eyes of their ophthalmic patients. From a practical point of view, the customary monocular visual acuity testing is often the first indication of deviating visual behaviour. Diagnostically, it should direct the attention to lesions of the chiasm. Further studies are clearly needed for a better understanding of what underlies the specific visual behaviour. 


\section{References}

[1] Fledelius, H.C. (2004) Chiasmal Pathology Causing Inability to Access Information in the Temporal Visual Field. European Journal of Ophthalmology, 28, 77-85. https://doi.org/10.1076/noph.28.2.77.23740

[2] Fledelius, H.C. (2009) Temporal Visual Field Defects Are Associated with Monocular Inattention in Chiasmal Pathology. Acta Ophthalmologica, 87, 769-775. https://doi.org/10.1111/j.1755-3768.2008.01328.x

[3] Fledelius, H.C. (2016) Cognitive Visual Disturbances in Chiasmal Disorders: A Review on Exceptional Visual Disabilities as Based on 30 Years' Clinical Experience. Journal of Medical \& Surgical Pathology, 1, 117.

[4] Glaser, J.S. (1999) Neuro-Ophthalmology. 3rd Edition, Lippincott, Williams \& Wilkins, Philadelphia, 17, 220.

[5] Samuelsson, H., Hjelmquist, E.K.E., Jensen, C. and Blomstrand, C. (2002) Search Pattern in a Verbally Reported Visual Scanning Test in Patients Showing Spatial Neglect. Journal of the International Neuropsychological Society, 8, 382-394. https://doi.org/10.1017/S1355617702813194

[6] Halligan, P.W., Cockburn, J. and Wilson, B.A. (1991) The Behavioural Assessment of Visual Neglect. Neuropsychological Rehabilitation, 1, 5-32. https://doi.org/10.1080/09602019108401377

[7] Marstrand, L. (2002) Cognitive Defects Associated with Posterior Cerebral Artery Infarctions. PhD Thesis, University of Copenhagen, Copenhagen.

[8] Mesulam, M. (1994) Higher Visual Functions of the Cerebral Cortex and Their Disruption in Clinical Practice. In: Albert, D.M. and Jacobiec, F.A., Eds., Principles and Practice of Ophthalmology, Saunders, Philadelphia, 2640-2653.

[9] Dutton, G.N. (2002) Cognitive Vision, Its Disorders and Differential Diagnosis in Adults and Children. Eye, 16, 1-17.

[10] Ringman, J.M., Saver, J.L., Woolson, R.E., Clarke, W.R. and Adams, H.P. (2004) Frequency, Risk Factors, Anatomy, and Course of Unilateral Neglect in an Acute Stroke Cohort. Neurology, 63, 468-474. https://doi.org/10.1212/01.WNL.0000133011.10689.CE

[11] Ptak, R. and Fellrath, J. (2013) Spatial Neglect and the Neural Coding of Attentional Priority. Neuroscience \& Biobehavioral Reviews, 37, 705-722.

https://doi.org/10.1016/j.neubiorev.2013.01.026

[12] Fledelius, H.C., Korsholm, K. and Law, I. (2015) Functional PET Scan in Four Patients with Higher Order Neglect-Like Cognitive Dysfunction Associated to Chiasm Related Pathology. Open Journal of Ophthalmology, 5, 54-60.

https://doi.org/10.4236/ojoph.2015.52009 
Submit or recommend next manuscript to SCIRP and we will provide best service for you:

Accepting pre-submission inquiries through Email, Facebook, LinkedIn, Twitter, etc. A wide selection of journals (inclusive of 9 subjects, more than 200 journals)

Providing 24-hour high-quality service

User-friendly online submission system

Fair and swift peer-review system

Efficient typesetting and proofreading procedure

Display of the result of downloads and visits, as well as the number of cited articles Maximum dissemination of your research work

Submit your manuscript at: http://papersubmission.scirp.org/

Or contact ojoph@scirp.org 